\title{
Localization and characterization of lymphatic vessels in oral and cervical squamous cell carcinoma
}

\author{
MASAHISA INOUE ${ }^{1}$, CHENG-HSIUNG ROAN ${ }^{3}$, TOMOMI ABE ${ }^{1}$, ROSARIO R. BUERY ${ }^{1}$, \\ HITOSHI NAGATSUKA ${ }^{2}$, NAOKI KATASE ${ }^{2}$, NORIYUKI NAGAI ${ }^{2}$ and KOJUN SETSU ${ }^{1}$ \\ ${ }^{1}$ Laboratories for Structure and Function Research, Faculty of Pharmaceutical Sciences, Tokushima Bunri University, \\ Tokushima; ${ }^{2}$ Department of Oral Pathology and Medicine, Graduate School of Medicine, Dentistry and Pharmaceutical \\ Sciences, Okayama University, Okayama, Japan; ${ }^{3}$ Tung's Taichung Metro Harbor Hospital, Taiwan, R.O.C.
}

Received April 1,2011; Accepted May 23, 2011

DOI: $10.3892 /$ etm.2011.277

\begin{abstract}
Lymph node metastasis is considered a factor in determining the prognosis of squamous cell carcinoma (SCC). Both oral and cervical SCC tumor cells prefer lymph vessels as the route of metastasis. D2-40 is a specific marker of lymphatic endothelial cells. This study clarifies the distribution and characteristics of lymphatic vessels in oral and cervical SCCs. Immunohistochemistry was performed in 20 oral and 20 cervical SCCs (10 non-metastatic and 10 metastatic to lymph nodes) using D2-40, CD31, CD34, CD105 and double staining with D2-40 and keratin. Lymphatic vessel density (LVD) was also determined morphologically. Results showed that lymphatic vessels in both types of SCCs were distributed mainly at the superficial region beneath the epithelium. The LVD in each tumor was significantly higher compared to the corresponding normal mucosa. Moreover, the LVD in lymph node metastasis in each tumor was significantly higher compared to their non-metastatic counterparts. Cancer cell invasion was observed in the lymphatic vessels suggesting the existence of lymph node involvement during metastasis. The new lymphatic vessels that proliferated around the cancer nests in both SCCs have endothelial cell characteristics inferred to be associated with early lymphatic development and initial dissemination of cancer cells.
\end{abstract}

\section{Introduction}

The role of blood vessels in tumor progression and metastasis has been reported in various malignancies, including oral and cervical cancers (1-3). However, the relationship between blood vessels and lymph node metastasis in malignant tumors

Correspondence to: Dr Masahisa Inoue, Laboratories for Structure and Function Research, Faculty of Pharmaceutical Science, Tokushima Bunri University, 180 Yamashiro-cho, Tokushima 770-8514, Japan

E-mail: inoue@ph.bunri-u.ac.jp

Key words: lymphatic vessel, squamous cell carcinoma, D2-40, CD34, CD105 is largely unknown. Angiogenesis plays a crucial role in hematogenous and lymphatic metastases for which studies have suggested that lesions that entered a higher angiogenic state have an increased probability of metastasis $(3,4)$. However, certain tumors with marked angiogenesis surprisingly had no evidence of metastasis and were associated with a good prognosis $(5,6)$. Thus, an unclear relationship exists among angiogenesis, metastasis and prognosis.

Clinically, lymph node metastasis in oral and cervical cancers occurs in 20-30\% of cases and is considered a major factor in determining prognosis. Lymphatic spread is more important than other routes. Via this route malignant cells preferentially metastasize in lymph nodes in the cervical region (7). Also, lymphatic vessels are the preferential routes for metastatic spread of most carcinomas that arise in the cervix (8). Distant lymph node metastasis of cancer cells is often due to the involvement of lymphatic vessels $(4,5)$. Despite the occurrence of lymphatic metastasis in those tumors, few studies have focused on the distribution and characteristics of lymphatic vessels in cancers $(9,10)$. Moreover, it remains questionable whether tumor cells induce lymphangiogenesis.

Several lymphatic-specific antibodies have been developed (11). Kahn and Marks developed an antibody called D2-40, reported to be effective in detecting lymphatic vessels in formalin-fixed paraffin sections (12). The antibody does not require any special treatment for antigen retrieval and so is considered to be useful in lymphatic research.

The aim of this study was to clarify the distribution of lymphatic tissues in oral and cervical squamous cell carcinoma (SCC) using immunohistochemistry. The study also elucidated the characteristics of lymphatic vessels involved in lymphangiogenesis.

\section{Materials and methods}

Case selection and tissue preparation. A total of 20 cases of oral SCC was acquired from the Department of Oral Pathology, Okayama University Hospital. Ten cases had lymph node metastasis and 10 cases were non-lymph node metastatic. Another 20 cases of cervical SCC from patients treated at Taipei Medical University were also included. Ten cases had lymph node metastasis and 10 were non-lymph 
Table I. Primary antibodies used.

\begin{tabular}{lcc}
\hline Antibody & Manufacturer & Dilution \\
\hline D2-40 & Dako & $1: 50$ \\
CD31 & Dako & $1: 100$ \\
CD34 & Nichirei & RTU \\
CD105 & Dako & $1: 50$ \\
Keratin & Dako & $1: 400$
\end{tabular}

RTU, ready to use.

A

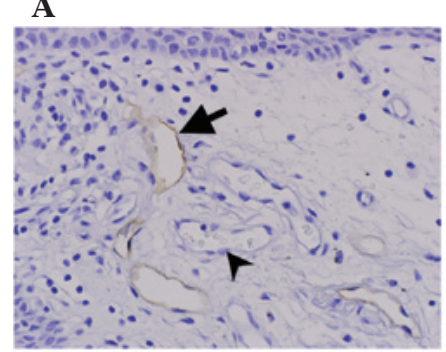

C

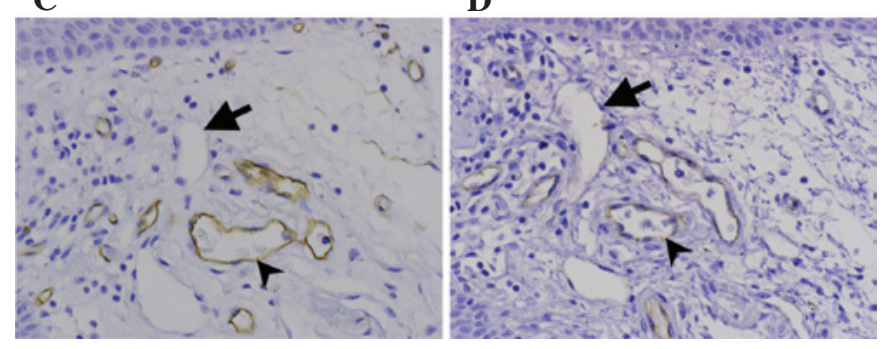

Figure 1. Staining patterns of (A) D2-40, (B) CD105, (C) CD34 and (D) CD31 in normal oral mucosa; lymphatic vessels (arrows), blood vessels (arrowheads); magnification, x200.

node metastatic. Five cases of each normal oral and cervical mucosal tissue were used as controls. The ethics committee of the university approved the study protocol. Conventional method for tissue preparation was performed where tissues were fixed in $10 \%$ neutral buffered formalin solution and embedded in paraffin. Serial sections $(4-\mu \mathrm{m})$ were prepared from paraffin blocks, stained with $\mathrm{H} \& \mathrm{E}$ and examined under a light microscope.

Immunohistochemistry. Table I shows the primary antibodies and their corresponding dilution. D2-40 is an antibody that detects lymphatic vessels and CD31, CD34, CD105 are antibodies against blood vessels. Keratin was used to identify SCC cells.

After deparaffinization, sections were immersed in $0.03 \%$ hydrogen peroxide in methanol for $30 \mathrm{~min}$. For CD31 and CD105, antigen retrieval was carried out by immersing the slides in $10 \mathrm{mM}$ sodium citrate buffer ( $\mathrm{pH}$ 6.0) and were highpressure heat treated at $121^{\circ} \mathrm{C}$ for $15 \mathrm{~min}$. Immunohistochemical staining was performed using the Vectastain ABC kit (Vector Laboratories, CA, USA). The antigen reaction was revealed using 3,3'-diaminobenzidine (DAB; Sigma-Aldrich, Tokyo,
A

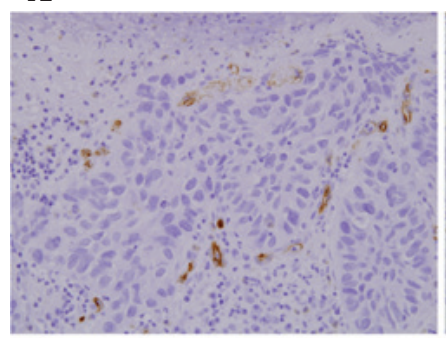

C

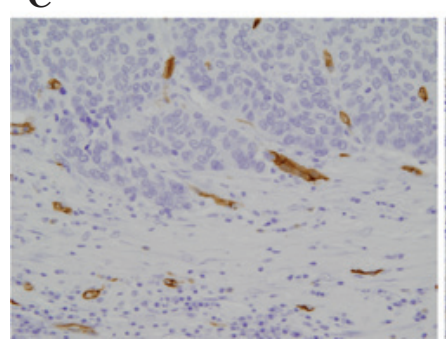

B

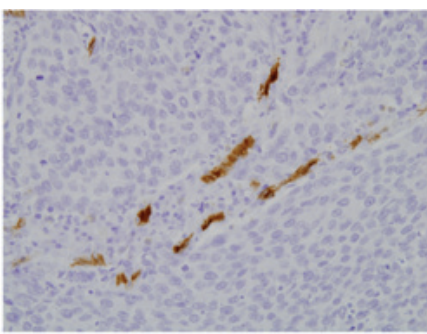

D

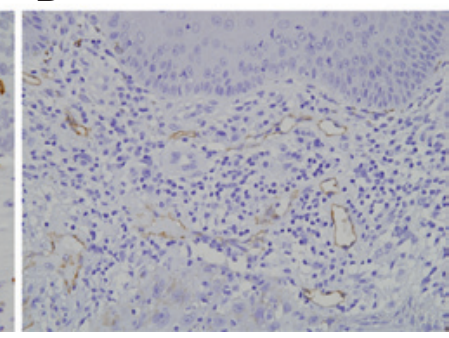

Figure 2. Immunolocalization in oral SCC. Numerous CD34-positive blood vessels were noted in the (A) superficial, (B) central and (C) deep regions. Most D2-40-positive lymphatic vessels were noted in the superficial region (D); magnification, $\mathrm{x} 200$.
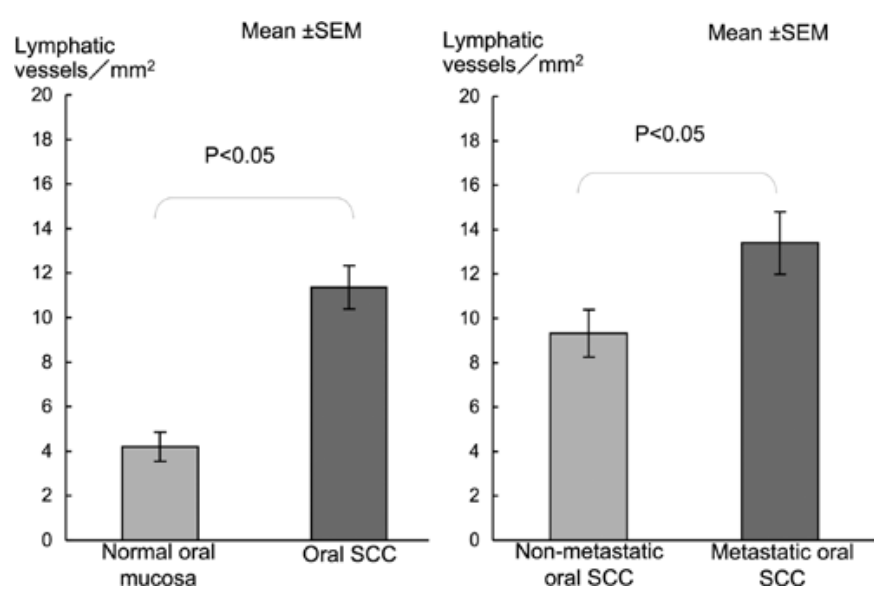

Figure 3. Comparison of mean LVD between normal oral mucosa and oral SCC and between non-metastatic and metastatic oral SCC.

Japan) chromogenic substrate, counterstained with Mayer's hematoxylin and examined under a light microscope.

To identify cancer cell invasion in lymphatic vessels, double staining was performed using keratin and D2-40 antibodies. For keratin, immunohistochemistry was performed using the Vectastain ABC kit (rabbit IgG; Vector Laboratories) followed by DAB chromogenic substrate. For D2-40, immunohistochemistry was carried out using the Vectastain ABC kit (mouse IgG; Vector Laboratories) and antigenic sites were revealed using 3-amino-9-ethylcarbazole (AEC; Dako, CA, USA). Sections were examined under a light microscope.

Lymphatic vessel density measurement and statistical analysis. Lymphatic vessel density (LVD) was measured by counting the number of lymphatic vessels stained with D2-40 on the superficial layer of normal mucosa as well as in the SCCs. Each sample was observed under a x20 objective $\left(\sim 0.54 \mathrm{~mm}^{2}\right)$, and five locations were chosen. Three different observers counted 
A

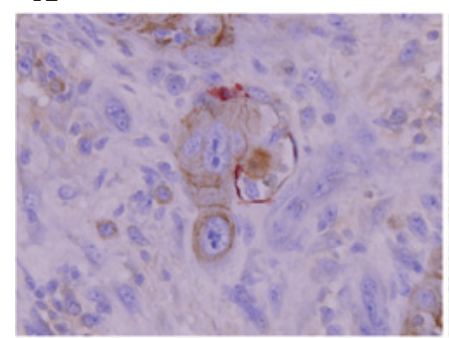

C

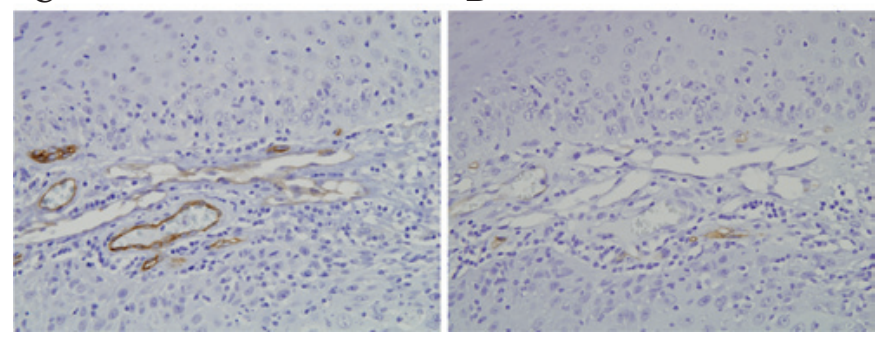

Figure 4. Characterization of tumor cells in oral SCC. (A) Double staining with D2-40/keratin showed lymphatic vessel infiltration of tumor cells; magnification, $x 400$. Several vessels were positive to (B) D2-40 and (C) CD105, but negative to CD34 (D); magnification, $x 200$.

A

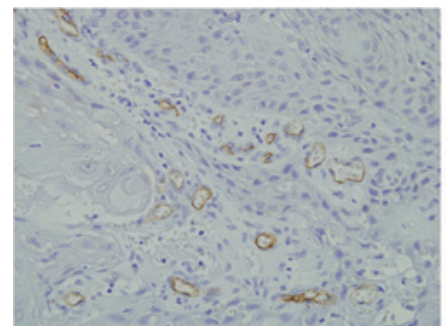

C

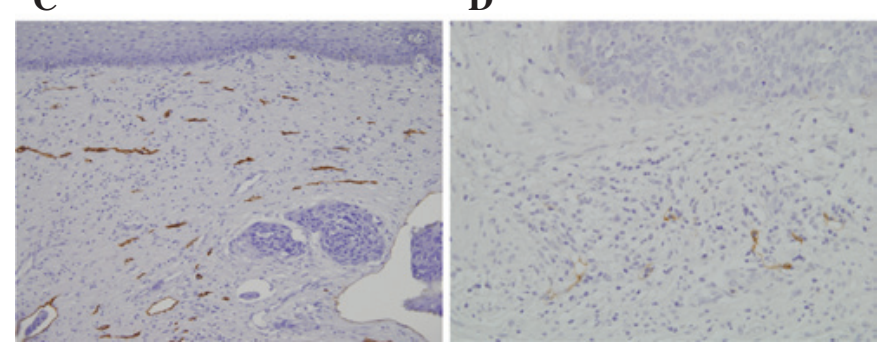

Figure 5. Immunolocalization in cervical SCC. Numerous CD34-positive blood vessels were noted in (A) superficial and (B) deep regions; magnification, x200. D2-40-positive lymphatic vessels were also observed in (C) superficial (magnification, $\mathrm{x} 50$ ) and (D) deep regions (magnification, $\mathrm{x} 200$ ).

the lymphatic vessels and then the average was computed. LVD was calculated per unit area $\left(1 \mathrm{~mm}^{2}\right)$.

\section{Results}

Distribution of lymphatic vessels in normal oral and cervical mисоsa. Lymphatic vessels in the oral and cervical mucosa stained with D2-40 in three consecutive tissue sections were examined as well as the surrounding blood vessels. In both oral and cervical mucosa, the blood vessels (arrowheads) were negative to D2-40 and CD105 (Fig. 1A and B) and positive to CD34 and CD31 (Fig. 1C and D). On the other hand, lymphatic vessels (arrows) were positive to D2-40 (Fig. 1A) and negative to
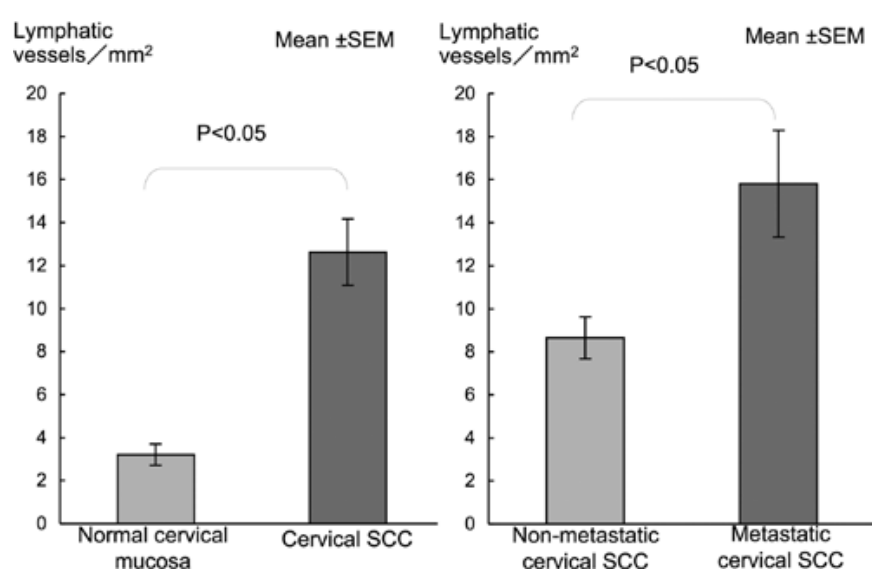

Figure 6. Comparison of mean LVD between normal cervical mucosa and cervical SCC and between non-metastatic and metastatic cervical SCC.

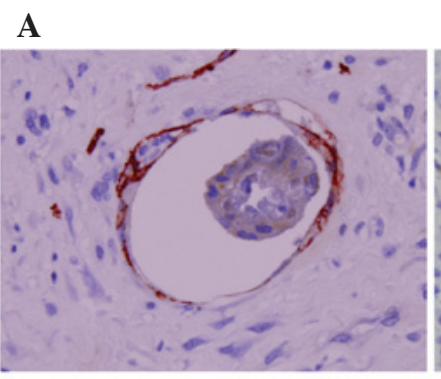

B

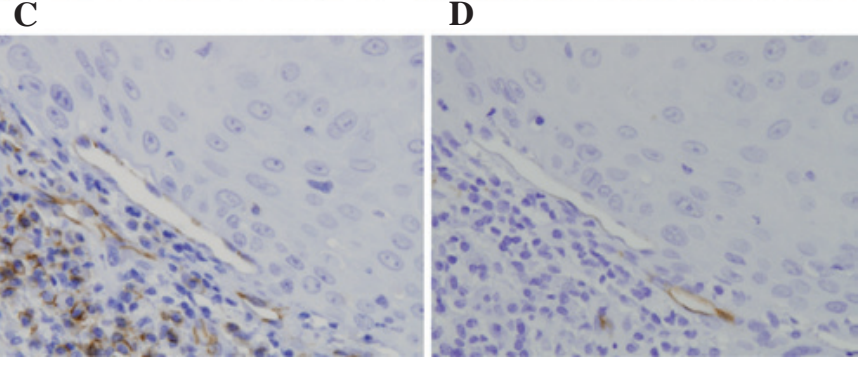

Figure 7. Characterization of tumor cells in cervical SCC. (A) Double staining with D2-40/keratin showed lymphatic vessel infiltration of tumor cells. Several vessels were positive to (B) D2-40 and (C) CD31, but negative to CD34 (D); magnification, $\mathrm{x} 400$.

CD105, CD34 and CD31 (Fig. 1B-D). Lymphatic vessels in the oral mucosa were distributed in the papillary and reticular layer (Fig. 1A). Lymphatic vessels in cervical mucosa were distributed in the reticular dermis and muscle layer (data not shown).

Localization and characterization of lymphatic vessels in oral SCC. Both metastatic and non-metastatic oral SCCs exhibited invasion of cancer cells in the connective tissue with proliferation of cancer nests. Blood vessels positive to CD34 were evenly distributed in the superficial cancer nests and invasive fronts (Fig. 2A-C). Lymphatic vessels positive to D2-40 were only localized in the superficial cancer nests (Fig. D). LVD in oral SCC was significantly higher than that in the normal mucosa (Fig. 3). LVD in metastatic SCC was significantly higher compared to that in the non-metastatic SCC (Fig. 3).

Double staining with D2-40 and keratin showed cancer cell infiltration in the lymphatic vessels (Fig. 4A) in a few 
non-metastatic and metastatic SCC. Cancer cell infiltration in lymphatic vessels was observed in 3 out of 10 metastatic SCC. Cancer cells were also observed around blood vessels. Notably, certain lymphatic vessels surrounding cancer nests were positive to D2-40 (Fig. 4B), CD31 (data not shown) and CD105 (Fig. 4C), but negative to CD34 (Fig. 4D).

Localization and characterization of lymphatic vessels in cervical SCC. Both non-metastatic and metastatic cervical SCC formed small nests, which invaded underneath the mucous membrane. In each serial section, blood vessels positive to CD34 were observed in the superficial and deep cancer nests showing the same degree of vascularity (Fig. 5A and B). Lymphatic vessels positive to D2-40 were localized in the superficial cancer nests (Fig. 5C). Scanty lymphatic vessels were noted in the central region and in the proliferating cancer nests (Fig. 5D). LVD in cervical SCC showed a significantly higher LVD than in normal mucosa (Fig. 6). A significantly higher LVD was noted in the metastatic compared to the nonmetastatic SCC cases (Fig. 6).

Double staining with D2-40 and keratin in both non-metastatic and metastatic SCC clearly showed infiltration of cancer cells in the lymphatic vessels (Fig. 7A). Cancer cell infiltration in lymph vessels was noted in 5 out of 10 metastatic cervical SCCs, which was almost the same with oral SCC. On the other hand, no cancer cells were observed in the blood vessels. Similar to oral SCC, lymphatic vessels around the cancer nests were positive to D2-40 (Fig. 7B), CD31 (Fig. 7C) and CD105 (data not shown), but negative to CD34 (Fig. 7D).

\section{Discussion}

Carcinomas preferentially metastasize via the lymph nodes. Clinical and pathological studies suggest that in many carcinomas, transport of tumor cells through the lymphatics is a common pathway of primary dissemination via afferent lymphatic vessels following the routes of natural drainage (8). The lymphatic system has many advantages compared to blood circulation thereby making it the preferred route of metastasizing tumor cells (13). Among these, lymph promotes cell viability since it is nearly identical to interstitial fluid without the serum toxins present in the blood. Furthermore, lymphatic vessels have low shear stress and mechanical deformation than blood vessels $(14,15)$. In addition, lymphatic vessels have thin walls lined by a layer of endothelial cells and discontinuous basement membrane. These features make lymphatic vessels optimally suited for entry and transport of cells (16). Hence, penetration and survival of metastasizing tumor cells is highly facilitated via lymphatic vessels.

In this study, lymphatic vessels expressing D2-40 were located at superficial cancer nests. LVD in the tumors were significantly higher than that in the normal mucosa. Moreover, LVD was significantly higher in cases with metastasis to the lymph nodes compared to the non-metastatic cases. These results suggest that tumor cells may have induced lymphangiogenesis during the initial or early stage in order to promote their initial dissemination. This may be the reason why oral SCC in particular involves the lymph nodes even at an early stage. As previously mentioned, lymphatic vessels provide a pathway conducive for the survival and dissemination of cancer cells.
Characterization of proliferating lymphatic vessels in SCC has not yet been elucidated. Lymphatic vessels surrounding cancer nests were positive to D2-40, CD31 and CD105, suggesting that the proliferating vessels associated with tumors have different biological characteristics than normal lymphatic vessels. The lumen of lymphatic capillaries is composed of a single layer of endothelial cells lining a thin part that overlaps each other and is known to have a discontinuous basement membrane unlike the immature capillary. The two theories on lymphangiogenesis include the centrifugal and centripetal theories (17). In the centrifugal theory, lymph sacs sprouting from venous endothelial cells occur early in life, spread to surrounding tissues and organs followed by budding endothelial cells forming around local lymphatic vessels. In the centripetal theory, lymphatic vessels originate by the fusion of flattened mesenchymal spaces into a primitive lymphatic network, which spreads integrally and then establishes a connection to the venous system. Both theories do not resolve the fundamental enigma of whether lymphatic differentiation originates from a primitive lymph sac from a vein or from a mesenchymal tissue space (18). Recent research has focused on the relationship between the differentiation of hematopoietic stem cells and endothelial cells (17). Lymphangiogenesis associated with tumor invasion is believed to arise by the growth of existing and budding from main lymphatic vessels (17). Studies have shown that lymphatic vessels during the embryonic stage are positive to CD31, and CD105 expression was noted during angiogenesis. New lymphatic vessels which proliferate around SCC are thought to revert back to a less differentiated form believed to have primitive characteristics of endothelial cells $(19,20)$, suggesting that lymphangiogenesis in cancer tissue supports the centrifugal theory.

The present study clarified the localization and characteristics of lymphatic vessels in oral and cervical SCCs. Lymphatic vessels in both SCCs were distributed mainly in the superficial region beneath the epithelium, and LVD was significantly higher in the SCCs than that in the normal mucosa. LVD in cases with lymph node metastasis was significantly higher compared to that in the non-metastatic SCC. Cancer cell infiltration in the lymphatic vessels was clearly observed suggesting the existence of lymph node involvement. The new lymphatic endothelial cells that proliferated around the cancer nests had primitive endothelial cell characteristics thought to be associated with early lymphatic development and initial dissemination of cancer cells.

\section{References}

1. Folkman J: Role of angiogenesis in tumor growth and metastasis. Semin Oncol 29: 15-18, 2002.

2. Abulafia O, Triest WE and Sherer DM: Angiogenesis in malignancies of the female genital tract. Gynecol Oncol 72: 220-231, 1999.

3. Nagatsuka H, Hibi K, Gunduz M, et al: Various immunostaining patterns of CD31, CD34 and endoglin and their relationship with lymph node metastasis in oral squamous cell carcinomas. J Oral Pathol Med 34: 70-76, 2005.

4. Weidner N, Semple JP, Welch WR and Folkman J: Tumor angiogenesis and metastasis-correlation in invasive breast carcinoma. N Engl J Med 342: 1-8, 1991.

5. Ranieri G, Labriola A, Achille G, et al: Microvessel density, mast cell density and thymidine phosphorylase expression in oral squamous cell carcinoma. Int J Oncol 21: 1317-1323, 2002. 
6. Hannen EJ, van der Laak JA, Manni JJ, et al: Computer assisted analysis of the microvasculature in metastasized and nonmetastasized squamous cell carcinomas of the tongue. Head Neck 24: 643-650, 2002

7. Zhang Z, Helman JI and Li LJ: Lymphangiogenesis, lymphatic endothelial cells and lymphatic metastasis in head and neck cancer - a review of mechanisms. Int J Oral Sci 2: 5-14, 2010.

8. Van Trappen PO and Pepper MS: Lymphangiogenesis in human gynaecological cancers. Angiogenesis 8: 137-145, 2005.

9. Agarwal B, Saxena R, Morimiya A, Mehrotra S and Badve S: Lymphangiogenesis does not occur in breast cancer. Am J Surg Pathol 29: 1449-1455, 2005

10. Xuan M, Fang YR, Wato M, Hata S and Tanaka A: Immunohistochemical co-localization of lymphatics and blood vessels in oral squamous cell carcinomas. J Oral Pathol Med 34: 334-339, 2005.

11. Marks A, Sutherland DR, Bailey D, et al: Characterization and distribution of an oncofetal antigen (M2A antigen) expressed on testicular germ cell tumours. Br J Cancer 80: 569-578, 1999.

12. Kahn HJ and Marks A: A new monoclonal antibody, D2-40, for detection of lymphatic invasion in primary tumors. Lab Invest 82: 1255-1257, 2002.

13. Pepper MS, Tille JC, Nisato R and Skobe M: Lymphangiogenesis and tumor metastasis. Cell Tissue Res 314: 167-177, 2003.
14. Liotta LA, Steeg PS and Stetler-Stevenson WG: Cancer metastasis and angiogenesis: an imbalance of positive and negative regulation. Cell 64: 327-336, 1991.

15. Weiss L and Schmid-Schönbein GW: Biomechanical interactions of cancer cells with the microvasculature during metastasis. Cell Biophys 14: 187-215, 1989.

16. Witte MH, Way DL, Witte CL and Bernas M: Lymphangiogenesis: mechanisms, significance and clinical implications. EXS 79: 65-112, 1997.

17. Kato S: Science of lymphangiogenesis. The Cell 37: 178-179, 2005.

18. Kotani M: The lymphatics and lymphoreticular tissues in relation to the action of sex hormones. Arch Histol Cytol 53: 1-76, 1990.

19. Wang JM, Kumar S, Pye D, van Agthoven AJ, Krupinski J and Hunter RD: A monoclonal antibody detects heterogeneity in vascular endothelium of tumours and normal tissues. Int $\mathrm{J}$ Cancer 54: 363-370, 1993.

20. Podgrabinska S, Braun P, Velasco P, Kloos B, Pepper MS and Skobe M: Molecular characterization of lymphatic endothelial cells. Proc Natl Acad Sci USA 99: 16069-16074, 2002. 